\title{
An Overview on Thermal Image Processing
}

\author{
Sheeba Ansari \\ Department of Electronics \& \\ Telecommunication \& Engineering \\ G H Raisoni College Of Engineering \\ Nagpur, India \\ Email: Sheeb12ansari@gmail.com
}

\author{
Prof. Suresh Salankar \\ Department of Electronics \& \\ Telecommunication \& Engineering \\ G H Raisoni College Of Engineering \\ Nagpur, India \\ Email: Suresh.salankar@raisoni.net
}

\begin{abstract}
Entire world is accentuated on inanition health certainty and food safety. Mostly, for fruit ripening the fruit seller uses calcium carbide and for human body the calcium carbide is exceptionally dangerous as it accommodates the phosphorous and arsenic traces. In many countries it is prohibited but in Pakistan, India, Nepal, and Bangladesh and in another country it is directly used. Quality assessment of banana fruit can be concluded by either human inspectors or instrumental tools. This paper presents a method of Thermal Imaging Technology for detection of banana fruit whether it is ripened by calcium carbide or naturally ripened. This paper also presents image preprocessing, image segmentation and feature extraction steps for processing of an image. For classifying these images the Neural Network is used.
\end{abstract}

Index Terms-Thermal Image; Banana Fruit; Neural Network; Feature Extraction; Segmentation.

\section{INTRODUCTION}

In the world bananas are the forth almost essential crops, India is the superior country for banana manufacturer. For food artifacts of high grade with increased assumptions and welfare standards, the requirement for correct, fast and equitable grade resolution of these attributes in food artifacts ongoing to grow [1]. Presently the fruits bananas are intentionally by chemicals being contaminated effecting health hazards. Randomly Toxic chemicals are used to ripen, grow and make banana fruits which act smooth fresher or last longer, especially during off season. Among the pretreatments, those are mainly resulted for banana fruits deliberated acceptance for best purchaser and ease better marketing, is synthetic banana fruit ripening [2].

The fruit seller of banana uses Calcium carbide for fruits ripening process. For human body the calcium carbide is really dangerous as it includes the arsenic and phosphorus traces. In numerous country of the world the calcium carbide is prohibited, but in India, Pakistan, Nepal, and Bangladesh it is directly used. Thus there is the short-term and long-term possibility of health effects eating banana fruits directly which are persuade to ripen. Calcium carbide has innumerable uses in steel industries, agriculture and chemical. It is colorless when unadulterated, but grayishwhite in color otherwise, small like-garlic fragrance. Undiscriminating pesticides on dissimilar variety of fruits conduct to effects of poisonous. Calcium carbide is indiscriminately used in partially to another advance practice of influencing fruit ripening like the banana fruits dipped in a solution of subjection of fruits to ethylene gas or the solution of ethrel/ethephon. The human health infects by using calcium carbide. The effect influences insensibility in the hands and legs, cold and damp skin, weakness and low blood pressure. The chemical remains inside the fruit could conduct miscarriage. Fruits which look delightful from upper coating may not be healthy for health. Fruits which have uniformly color, example, a dozen of bananas having a uniformly color, are further have been ripened artificially [2].

It is an exacting problem to detect contamination in fruit that are generally take place underneath the skin of fruit. Detection defects furthermore considerably affected by numerous factors like time, contamination type, contamination extremity, fruit difference, and fruit pre- and postharvest states [3]. The fruits which are ripened naturally are not yellow uniformly; preferably, they are of green and yellow. Whenever mango and papaya are constantly orange/ yellow or tomatoes are red, then fruit sellers may have been used Calcium carbide; banana fruits can be identified if the stem is dark green where the remaining portion of banana fruits are yellow [2]. To determine such kind of problem in fruits Thermal imaging Technique is used.

\section{A. Thermal Imaging}

The thermal imaging technology detects the heat given off by an object or a person. The use of thermal imaging is to detecting infrared radiation means the heat source and generates the electrical signals from these laser signals, and provides the images in the form of heat. In medical imaging this technology has been widely used, this technique also used in, fault diagnosis, non-destructive testing and structure defect detection. This type of technology can instantly diagnostic target envision, rapid hot spots points, and also thermal profile verification, to regulate the problem condition [4]. Now this technique will be used to detect whether the banana fruit is contaminated or de-contaminated by toxic chemicals (Calcium carbide) or not. Because for ripening a fruit calcium carbide have been extensively used.

The rest of the paper is organized as follows. Section II describes the related work, section III presents the proposed work and in section IV we conclude our paper. 


\section{Related Work}

In [4] the thermal imaging is used for observing and fault diagnosis in electrical equipment. They used thermal camera for images of electrical equipment in experiments of nonidentical conditions, after that contacting with noise denoising, for image processing the segmentation and feature extraction is used, and then finally the analysis of image is generalized by using algorithms of artificial intelligence and check whether there is fault or not. In this the threshold segmentation technology is most widely used as compared to the detection edge segmentation or the segmentation method based on region. In electrical equipment's image they detect the abnormal heating condition. In feature extraction they extricate the images which were considered as fault, features those indicates the important characteristics and ancient. Ancient characteristics refers an image characteristics which is perfectly not weak and does not depend on environment conditions. In Intelligent fault diagnosis methods they used neural network methods.

In [5] they used an Electronic based nose system, which recruit an array of in exorbitant trading tin-oxide fragrance sensors, have been used to analyze ripeness state of banana fruit. To define seven different regions in multisensory space according to the ripeness state of bananas an investigatory techniques and principal component analysis were used, to estimate the banana-skin colors from classification. In equipment of electronic-nose, the signals generated by sensors are organized such a pattern-recognition engine that permits system to analyze multiplex aroma. Neural Network has been used extensively to perform pattern-recognition. Today back-propagation-trained multilayer perceptron (MLP) paradigm is the more accepted pattern-recognition step in aroma inspection. Although, there is problem in aroma classification, because in some instance the Fuzzy ARTMAP paradigm outperforms MLP. Optimistic technique is another learning vector quantization (LVQ) technique, it is supervised technique which is based on (SOM) selforganizing paradigm.

In [6] a thermal imaging technique is used for (PCB) Printed Circuit Board and their analysis is done using MATLAB. They have been taken a series of 20 thermal images which are stored in form of .png. These images are of the identical PCB in various conditions of thermal load. Particularly each image corresponds electrical operation. To aid the different characteristics of image analysis they also develop a tool based on MATLAB. Maximum area of peak temperature and the peak temperature variables are used for analysis those corresponds the regions with heat dissipation. MATLAB based tool is used to analyze all the images which are related to PCB and on the basis of that they also drawn useful conclusion accurately in much lesser time. On the basis of MATLAB the Graphical User Interface (GUI) is used for designer to choose censorious image to see temperature jump in between two images which are appreciably various temperature profiles. They used clustering based segmentation methods to select the region of interest should be permitted. For processing of thermal image of PCB's the standard methodologies of image processing are uses like color-based segmentation, histogram thresholding technique, image production difference, image into video conversion and histogram comparisons.

In [7] they discuss about the Infrared thermography or thermal imaging which is very appropriate, flexible and noncontact method used for many types of manual benefit like as electronic component, building surveys, and mechanical component but this technology nowadays used in machine conditioning observing such as fault detection, identification and resemblance for fault diagnosis. This paper represents a survey on various thermal imaging techniques for fault diagnosis and detection is based on the temperature of object. Image Histogram and Image Filtering techniques are used for thermal image inspection. The method for contrast enhancement and actual edge filtering is based on statistical differencing, where each pixel value is ascend by standard deviation and by using this they classify different machine conditions of thermal image. Feature such as standard deviation, mean, energy, and entropy are extracted from an image. The method used for classification of an image and machine condition diagnosis is Artificial Neural Network.

In [8] they discussed about the external grade of vegetables and fruits by different system of computer vision. The most common system of computer vision for externally grade assessment is the system of traditional computer vision on the basis of video cameras in the form of RGB which emulate the human eye vision which capture images by filters concentrate at RGB wavelengths. By using the traditional computer vision system most external grade attributes like size, color, shape, texture, and other obvious defects which can be deliberated, sometimes for unobvious defects the detection done by traditional computer vision system is unsustainable /demanding in normal color images because of insufficiency of multi-constituent information and spectral information. By using the devices of dispersive of wavelength, the cameras of high resolution, and recently approach in software computer and hardware computer, the computer vision system like hyper spectral and multispectral have been evolved and well organized examination equipment of agriculture artifacts for the safety and grade of diversity. For external grade feature of vegetable and fruit, Plenty of prosperous applications have proven that hyper spectral and multispectral computer vision systems are excellent equipments and also for bruise, unobvious defect and chilling injury assessment.

In [1] they discussed that bananas is retailed in bunch of dozen so it is essential to analyze grade in bunch. The convenient algorithm can measure systematically the grade of one banana but if it is apply on dozen of banana the setup for acquisition of an image are not train of algorithm for interpretations are undignified. On the basis of review they can say that acquisition of an image is an essential method all over another method. The texture and color features integration provides leading result in the process of classification of banana. They choose selected features those can be associated with attributes of purchaser, and attributes 
of bananas. To recognize levels of bananas they evolved statistical model and also contraption the system for processing of an image for bananas classification by extracting feature.

In [9] Image processing technique is used to calculating the ethylene gas for ripening of fruits. In practice they also survey on existing methods for fruits ripening. From this it is recognized that exact concentration of ethylene gas under supervised temperature and pressure can be used for ripening process which is contemplated as a secure process. The color image processing is used to find the application in this area to calculate the carbon dioxide gas levels and ethylene gas levels with respect to the color of fruit.

In [10] they use Neural Network for classification and identification of various varieties of bulk fruit images. For feature extraction the texture and color features are extricated contemplating the entire image. The features which are extricated are saved in knowledge based form. If new image is experienced they extricated feature from the sample of fruit image. Neural Network is used to classify and identify the features which are extricated.

In [11], [12], and [13] they used different machine learning technique such as ANN, SVM, KNN, and DNN for classification of data after selecting the feature extraction. In this paper the Artificial Neural Network technique is used for classifying the images and features are extricated to identify the images.

From above literature, it is concluded that the thermal imaging is used to detect the fault diagnosis and machine conditioning observations. Thermal imaging techniques for fault diagnosis and detection are based on the temperature of object [3], [4], [6] and [8]. In [5] the system based on Electronic nose, which recruit an array of in exorbitant trading fragrance sensors for tin-oxide, used to analyze the ripeness state of banana fruit. In [8] the computer vision system like multispectral and hyper spectral computer vision systems are excellent equipments in vegetable and fruit external grade feature, particularly for bruise, unobvious defect and chilling injury assessment as compare to system like traditional vision computer. In [1] banana classification and quality analysis is done using image processing technique and quality relates to ripening stages of banana but not for the quality for contamination or decontamination with toxic chemicals. In [9] they conclude that ripening process by using exact concentration of ethylene gas is considered as a safe process. Image processing is used for ethylene gas measurement for fruit ripening. For ripening process the Calcium carbide is also used but the detection of Calcium carbide in fruit was not identified.

\section{Proposed Work}

This paper presents an overview on Thermal Imaging Technique for detection of fruit whether it is contaminated or de-contaminated by toxic chemicals (Calcium carbide) or not. The image is captured by Thermal Imaging Camera FLIR (ThermaCam E45). Thermal camera detects the defects in fruits. If fruit is ripened by calcium carbide means it is contaminated by calcium carbide and when image of that banana fruit is taken by thermal camera then it represents the information of an image in terms of temperature. The different temperature profile for each sample will be generated by thermal camera and classification of an image is done by using artificial neural network. Image is processing mainly in five steps as follow

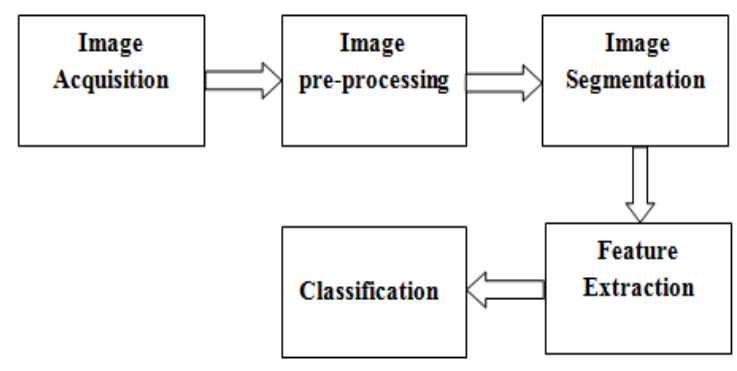

Fig. 1 Process of Classification of Banana

Pre-processing, Classification, and feature extraction are the three main processes in image processing.

\section{Step 1: Pre-processing}

Pre-processing is the first step for the conversion of thermal image into gray scale image which is followed by filtering process and resizing of image to eliminate unwanted part of the banana fruit.

\section{A. Image Filtering}

In this paper for filtering of images the wiener and median filter is used. Median filter produces good result as compared to wiener filter. So median filter is use for filtering process.

\section{B. Median Filter}

Nonlinear median filtering is used to eliminate noise from an image. It is very productive so it is used to eliminating noise to conserving edges. It is extremely productive to eliminating noise like pepper and salt. The working of median filter is done by affecting across the pixel image through pixel, exchanging each value with neighboring median pixels value. The design of neighbors is known as "window", which glides, pixel by pixel overall the whole image. Image median is estimated by firstly categorize all pixel values into numerical sequence from window and after that pixel value which is actually accounted with the median pixel value. The input pixel which is exchanged by middle of the pixel is incorporate around the pixel in the window.

The median filtering algorithm instructs in window arrange the pixel values in increasing or decreasing sequence and selecting the median value of the image. Median is calculated by in the middle by two average values. Typical windows are $5 \times 5,7 \times 7,3 \times 3$, or the 5 point window is reviewed for special type of averaging. 


\section{Image Enhancement}

To highlighting, or improving, the feature of an image like contrast or boundaries, detection of an edge an image enhancement is used to make a visual display which is most applicable for analysis and analysis. The process of enhancement increases the vital range of selecting feature so that they can be easily detected. Image enhancement included contrast manipulation and gray level, crispening edge and noise depletion, improving, psudocoloring, filtering, magnification insertion etc. image enhancement is a very important topic because of its usefulness essentially in all image processing application. There are some much techniques for image enhancement such as contrast enhancement restricted adaptive histogram equalization. In this paper for image enhancement of banana fruit the histogram equalization is used.

\section{Step 2: Image Segmentation}

In image processing the important step is the image segmentation and it is exacting step position to separate the image constituent regions of interest or area. Segmentation methods are of four types namely classification-based segmentation, edge-based segmentation, threshold-based segmentation and region-based segmentation. The analysis and processing of an image depends on segmentation of an image. In this paper the color based segmentation is used to segment the contaminated portion in banana fruit. It is attainable to segment image on the basis of color. This can be done by situating of object based on color in an image is attainable. Analysis of image can be accomplished either straightly on the true image or either across individual color of plane. On the basis of intensity value of color to segment the image the threshold is set because intensity values are sustained in homogeneous color region. Specific color range with an object can be detected easily from whole image. The mean value of necessitate color is calculated and it is compared with each RGB pixels values of an image using either Mahalanobis distance measure or the Euclidian distance measure. The particle swam optimization (PSO) algorithm is used for color based segmentation.

\section{Step 3: Feature Extraction}

It is the important step in image processing. The most important aim is to extricate the feature from an image. For feature extraction we evaluate the area of an object (i.e. area of banana fruit) in binary image. An input images either a numeric or logical. If an input is numeric then nonzero pixels are appraised like Area, Mean, Standard Deviation, min and max values of RGB. These features are calculated from the images because all the extricated features having different values for different thermal images and on the basis of these parameters the normal and contaminated images can also be classified.

\section{Step 4: Classification}

After feature extraction the images will be classified by using Artificial Neural Network. The determined features from an image used as inputs, i.e. number of inputs nodes are identical to required number of features. In the first hidden layer number of neurons can be uniform or lesser than number of characteristics in classification. Artificial neural network can also have user-defined following hidden layers and these permit the nonlinear additional organizing of input attributes. The artificial neural network is not the linear system, like the method permits extra decoration correlate and data diminution. Bananas are classified based on all factors values and parts.

\section{Conclusion}

The accurately identification and classification of banana fruit whether it is ripened by calcium carbide or naturally ripened is very important for human body as calcium carbide accommodates traces of arsenic and phosphorous. This paper presents a method of Thermal Imaging Technology for detecting such kind of problem because different techniques are used to detect the quality of fruits and in this paper, we focused on the contamination of fruit by toxic chemicals or not. Different steps for image processing are also discussed and Neural Network is used for classification purpose for image.

\section{REFERENCES}

[1] Sanjay Chaudhary, Bhavesh Prajapati, "Quality Analysis and Classification of Bananas" International Journal of Advanced Research in Computer Science and Software Engineering Volume 4, Issue 1, January 2014

[2] Md. Wasim Siddiqui* and R.S Dhana "Eating artificially ripened fruits is harmful" General Article CURRENT SCIENCE.VOL.99, No. 12, 25 December 2010

[3] Zhou Jianmin, Zhou Qixian, Liu Juanjuan, Xu Dongdong, "Design of on-line detection system for apple early bruise based on thermal properties analysis" International Conference on Intelligent Computation Technology and Automation 2010.

[4] Haoyang Cui, Yongpeng $\mathrm{Xu}$, Jundong Zeng and Zhong Tang, "The Methods in Infrared Thermal Imaging Diagnosis Technology of Power Equipment" 2013 IEEE.

[5] Eduard Llobet, Evor L Hines, Julian W Gardner, and Stefano Franco, "Non-destructive banana ripeness determination using a neural network-based electronic nose" IOP publishing Ltd 26 March 1999.

[6] Jibu Varghese k, Tripty Singh, sreyas Mohan, "PCB Thermal Image Analysis using MATLAB" ISSN (Online): 2347 - 2812, Volume-2, Issue - 3, 2014.

[7] Ashish, Vijay, "Review on Thermal Image Processing Techniques for Machine Condition Monitoring" international Journal of Wireless Communications and Networking Technologies Volume 3, No.3, April-May 2014.

[8] Baohua Zhang, Wenqian Huang....etc. Principles, developments and applications of computer vision for external quality inspection of fruits and vegetables: A review. Food Research International 62(2014) 326-343.

[9] V. Srividhya, K. Sujhata and R.S. Ponmagal, "Ethylene Gas Measurement for Ripening of Fruits Using Image Processing" Indian Journal of Science and Technology, Vol 9(31), August 2016.

[10] Dayanand Savarkar "Identification and Classification of Bulk Fruits Images using Artificial Neural Networks" IJEIT Volume 1, Issue 3, March 2012.

[11] Semwal, Vijay Bhaskar, Kaushik Mondal, and G.C. Nandi. "Robust and accurate feature selection for humanoid push recovery and classification : deep learning approach." Neural Computing and Applications (2015): 1-10

[12] Semwal, V.B., Singha, J., Sharma, P.et al., "An optimized feature selection techniques based on incremental feature analysis for biometric gait data classification" Multimed Tools App (2016). doi: 10.1007/s 11042-016-4110-y

[13] Semwal, Vijay Bhaskar, Manish Raj, and Gora Chand Nandi. "Biometric gait identification based on a multilayer perceptron ." Robotics and Autonomous Systems 65 (2015): 65-75. 